\title{
A Random Matrix Models of Relaxation
}

\author{
J. L. Lebowitz \\ Department of Mathematics, Rutgers University, USA * \\ L. Pastur \\ Department of Mathematics, University Paris 7, France ${ }^{\dagger}$
}

\begin{abstract}
We consider a two level system, $\mathcal{S}_{2}$, coupled to a general $n$ level system, $\mathcal{S}_{n}$, via a random matrix. We derive an integral representation for the mean reduced density matrix $\rho(t)$ of $\mathcal{S}_{2}$ in the limit $n \rightarrow \infty$, and we identify a model of $\mathcal{S}_{n}$ which possesses some of the properties expected for macroscopic thermal reservoirs. In particular, it yields the Gibbs form for $\rho(\infty)$. We consider also an analog of the van Hove limit and obtain a master equation (Markov dynamics) for the evolution of $\rho(t)$ on an appropriate time scale.
\end{abstract}

PASC numbers: $\quad$ 05.30.Ch, 05.60.Gg, 05.70.Ln

\section{Introduction}

The time evolution of a "small" quantum system interacting with a "big" thermal reservoir is a much studied problem in various contexts, see review works [9, 7, 3, 11, 17, 6, 1,. Two natural questions for such models are the large time limit of the state of the small system and the nature of the approach to this limit. It is generally expected and rigorously proven for certain types of reservoirs and couplings that, starting from an arbitrary initial state of the system and an equilibrium Gibbs state at temperature $T$ of the reservoir, the final, $t \rightarrow \infty$, state of the system is given by a projection of the joint system plus reservoir Gibbs measure at the temperature $T[1,6]$.

In this paper we discuss the above questions in the frameworks of random matrix theory. Namely, we consider a small system $\mathcal{S}_{2}$, having only two levels (like in the archetype spin-boson model [17, 1, 6]), coupled to a system with $n$ levels (denoted by $\mathcal{S}_{n}$ ) via an $n \times n$ random matrix. Similar models have been discussed in papers [2, 14, 16, 12. Here we will investigate in a more explicit way what features of the $n$-level system will lead, in the limit $n \rightarrow \infty$, to the reduced density matrix $\rho(t)$ of the small system having a Gibbs form for large times and whether $\rho(t)$ is described by a Markov evolution. Our analysis will be based entirely on the fact that the interaction is given by a typical realization of a random matrix. This models in some way a strongly interacting reservoir.

The paper is organized as follows. In Section 2 we describe the model and define the reduced density matrix for the small, two level, system. Since it is random, its full

*Also Department of Physics of Rutgers University

$\dagger$ Also Mathematical Division, Institute for Low Temperature Physics, Kharkov, Ukraine 
statistical description is of interest. In this paper we confine ourselves to the analysis of its mean which we argue is also its typical behavior in certain cases. In Section 3 we consider the equilibrium properties of the combined system. We find the reduced density matrices of $\mathcal{S}_{2}$ corresponding to the microcanonical and canonical description of the composite system and give conditions for their equivalence.

Section 4 deals with time dependent (non-equilibrium) properties of the model. We find that the $t \rightarrow \infty$ limit of $\rho(t)$ depends in general on the initial density matrix. However, for the reservoir introduced in Section 3 designed to guarantee the equivalence of the microcanonical and canonical description in equilibrium, the $t \rightarrow \infty$ limit of the diagonal entries of the mean reduced density matrix is independent of the initial density matrix and has the Gibbs form. In Section 5 we treat the van Hove asymptotic regime (small coupling, long times). Here both the equilibrium and the time dependent form of the mean reduced density matrix can be expressed directly via the density of states of the reservoir. We find that for the special form of the density of states of $\mathcal{S}_{n}$ of Section 3 the $t \rightarrow \infty$ limit of $\rho(t)$ is the Gibbs distribution, and its time evolution is Markovian. However, in this case the obtained time decay rates prove to be vanishing. In Section 6 we consider a more general model, where one can obtain quite reasonable decay rates. The model is similar to that, studied in [12]. In Section 7 we continue studies of the time evolution $\rho(t)$ discussing its dependence on the initial density matrix and on the parameters. Several formulas, used in the main body of the paper, are derived in Appendices.

\section{Description of Model}

Let $h_{n}$ be a real symmetric $n \times n$ matrix with eigenvalues $E_{j}, j=1, \ldots, n$. We characterize the spectrum of $h_{n}$ by its density of states

$$
\nu_{0}^{(n)}(E)=n^{-1} \sum_{j=1}^{n} \delta\left(E-E_{j}\right) .
$$

We assume that $\nu_{0}^{(n)}$ converges as $n \rightarrow \infty$ to a limiting density $\nu_{0}$, i.e. that for any continuous and rapidly decaying function $\varphi$ we have:

$$
\lim _{n \rightarrow \infty} \int_{-\infty}^{\infty} \varphi(E) \nu_{0}^{(n)}(E) d E=\int_{-\infty}^{\infty} \varphi(E) \nu_{0}(E) d E, \quad \int_{-\infty}^{\infty} \nu_{0}(E) d E=1 .
$$

Let $w_{n}$ be a real symmetric $n \times n$ random matrix, whose probability density is

$$
Q_{n}^{-1} \exp \left\{-\frac{1}{4} \operatorname{Tr} w^{2}\right\},
$$

where $Q_{n}$ is the normalization constant. In other words, the entries $w_{j k}, 1 \leq j \leq k \leq n$ of the matrix $w_{n}$ are independent Gaussian random variables, with

$$
\left\langle w_{j k}\right\rangle=0, \quad\left\langle w_{j k}^{2}\right\rangle=\left(1+\delta_{j k}\right),
$$

where the symbol $\langle\ldots\rangle$ denotes averaging with respect to the distribution (2.3). This probability distribution is known as the Gaussian Orthogonal Ensemble (GOE) [13]. 
We define the Hamiltonian of our composite system $\mathcal{S}_{2, n}$ as a random $2 n \times 2 n$ matrix of the form

$$
H_{n}=s \sigma^{z} \otimes \mathbf{1}_{n}+\mathbf{1}_{2} \otimes h_{n}+v \sigma^{x} \otimes w_{n} / n^{1 / 2},
$$

where $\mathbf{1}_{l}(l=2, n)$ is the $l \times l$ unit matrix, $\sigma^{z}$, and $\sigma^{x}$ are the Pauli matrices

$$
\sigma^{x}=\left(\begin{array}{ll}
0 & 1 \\
1 & 0
\end{array}\right), \sigma^{z}=\left(\begin{array}{ll}
1 & 0 \\
0 & -1
\end{array}\right) .
$$

The symbol $\otimes$ denotes the tensor product, and $s$ and $v$ are positive constants.

The first term in (2.5) is the Hamiltonian of $\mathcal{S}_{2}$, the second term is the Hamiltonian of $\mathcal{S}_{n}$, and the third term is an interaction between them. Thus $s$ determines the energy scale of the isolated small system, and $v$ plays the role of the coupling constant between the two level system $\mathcal{S}_{2}$ and the $n$ level system $\mathcal{S}_{n}$. Note also the $n^{-1 / 2}$ scaling of the interaction.

We emphasize that letting $n \rightarrow \infty$ does not imply that $\mathcal{S}_{n}$ will behave like a macroscopic system. $\mathcal{S}_{n}$ could perfectly well be just a single particle moving on a periodic chain of $n$ sites with spacing $\delta$, for which, in the limit $n \rightarrow \infty$,

$$
\nu_{0}(E)=\left(\pi \sqrt{E\left(4 \delta^{-2}-E\right)}\right)^{-1}, 0<E<4 \delta^{-2} .
$$

In fact the normalization assumption (2.2) implies that we are not dealing here with what one usually thinks of as a thermal reservoir.

We are going to study the time evolution of the mean reduced density matrix of $\mathcal{S}_{2}$, assuming that at $t=0$ the density matrix of the composite system $\mathcal{S}_{2, n}$ is of the form

$$
\mu_{m}^{(n)}(0)=\rho(0) \otimes P_{k}
$$

where $\rho(0)$ is a $2 \times 2$ positive definite matrix of unit trace and $P_{k}$ is the projection on the state of energy $E_{k}$ of the reservoir. Let

$$
\mu^{(n)}(t)=\left\{\mu_{\alpha j, \beta k}^{(n)}(t), \alpha, \beta= \pm, j, k=1, \ldots, n\right\}
$$

be the density matrix of the composite system $\mathcal{S}_{2, n}$ at time $t$, corresponding to the initial density matrix $\mu_{m}^{(n)}(0)$ of (2.7). Then the mean reduced density matrix of the small system is defined as

$$
\rho_{\alpha, \delta}^{(n)}\left(E_{k}, t\right)=\sum_{j=1}^{n}\left\langle\mu_{\alpha j, \delta j}^{(n)}(t)\right\rangle=\sum_{j=1}^{n}\left\langle\left(e^{-i t H_{n}} \mu_{m}^{(n)}(0) e^{i t H_{n}}\right)_{\alpha j, \delta j}\right\rangle .
$$

We have evidently for all $k=1, \ldots, n$ and $t \geq 0$ :

$$
\sum_{\alpha= \pm} \rho_{\alpha, \alpha}^{(n)}\left(E_{k}, t\right)=1
$$

The mean reduced density matrix is determined by the initial density matrix $\rho(0)$ of $\mathcal{S}_{2}$ via the linear relation

$$
\rho_{\alpha, \delta}^{(n)}\left(E_{k}, t\right)=\sum_{\beta, \gamma= \pm} T_{\alpha \beta \gamma \delta}^{(n)}\left(E_{k}, t\right) \rho_{\beta, \gamma}(0)
$$


where

$$
T_{\alpha \beta \gamma \delta}^{(n)}\left(E_{k}, t\right)=\sum_{j=1}^{n}\left\langle\left(e^{-i t H_{n}}\right)_{\gamma k, \delta j}\left(e^{i t H_{n}}\right)_{\alpha j, \beta k}\right\rangle,
$$

is the "transfer" matrix. It can be viewed as an analogue of the influence functional, introduced by Feynman and Vernon in a similar context [5].

A particular case of the mean reduced density matrix is the mean transition probability $p_{\alpha, \delta}^{(n)}(E, t)$ for $\mathcal{S}_{2}$ to be found in the state $|\alpha\rangle$ at time $t$ provided that at $t=0$ it was in the state $|\beta\rangle$ and the initial state of $\mathcal{S}_{n}$ was the pure state of energy $E_{k}$ for some $k$. It is given by

$$
p_{\alpha, \delta}^{(n)}\left(E_{k}, t\right)=T_{\alpha \delta \delta \alpha}^{(n)}\left(E_{k}, t\right)=\sum_{j=1}^{n}\left\langle\left|\left(e^{-i t H_{n}}\right)_{\alpha j, \delta k}\right|^{2}\right\rangle,
$$

with

$$
\sum_{\alpha= \pm} p_{\alpha, \delta}^{(n)}\left(E_{k}, t\right)=1, \delta= \pm .
$$

The diagonal entries $p_{\alpha, \alpha}^{(n)}\left(E_{k}, t\right), \alpha= \pm$ are known as survival (or return) probabilities.

We can evidently replace $P_{k}$ by any superposition $\sum_{k} c_{k} P_{k}$ of pure states of $\mathcal{S}_{n}$, e.g. by that, corresponding to the canonical density matrix $e^{-\beta h_{n}} / Z_{n}$, where $Z_{n}$ is the partition function of $\mathcal{S}_{n}$ and $\beta$ is its inverse temperature. The initial density matrix for the composite system will now be (cf (2.7) $)$

$$
\mu_{c}^{(n)}(0)=\rho(0) \otimes e^{-\beta h_{n}} / Z_{n}
$$

corresponding to the choice

$$
c_{k}=e^{-\beta E_{k}} / n \int_{-\infty}^{\infty} e^{-\beta E} \nu_{0}^{(n)}(E) d E,
$$

where $\nu_{0}^{(n)}$ is defined in (2.1).

It should be mentioned, that our results remain valid also in the case, where the matrix $h_{n}$ is non-diagonal, random, independent of $w_{n}$, and possesses property (2.2) with probability 1. However, the randomness of $h_{n}$ plays practically no role. Besides, our results are not limited to the special Gaussian distribution (2.3) - (2.4) of the interaction, but hold also for any real symmetric random matrix $w_{n}$ in (2.5), whose entries $w_{j k}, 1 \leq$ $j \leq k \leq n$ are independent, verify (2.4) and have fourth moments bounded uniformly in $n$. The techniques in this case are, however, more involved.

\section{Equilibrium States}

\subsection{General formulas.}

We begin by considering the equilibrium (time independent) microcanonical density matrix of the composite system $\mathcal{S}_{2, n}$ :

$$
\Omega(\lambda)=\delta\left(\lambda-H_{n}\right) / \operatorname{Tr} \delta\left(\lambda-H_{n}\right)
$$

Following a standard prescription of statistical mechanics, we will replace the Dirac deltafunction in (3.1) by the function $(2 \varepsilon)^{-1} \chi_{\varepsilon}$, where $\chi_{\varepsilon}$ is the indicator of the interval $(-\varepsilon, \varepsilon)$, 
and $\varepsilon \ll \lambda$. Then the reduced microcanonical density matrix of $\mathcal{S}_{2}$, i.e., the microcanonical density matrix of $\mathcal{S}_{2, n}$, traced with respect to the states of $\mathcal{S}_{n}$, is the $2 \times 2$ matrix of the form

$$
\omega^{(n)}(\lambda)=\frac{\bar{\nu}^{(n)}(\lambda)}{\sum_{\delta= \pm} \bar{\nu}_{\delta, \delta}^{(n)}(\lambda)}
$$

where

$$
\bar{\nu}_{\alpha \gamma}^{(n)}(\lambda)=(2 \varepsilon n)^{-1} \sum_{j=1}^{n} \chi_{\varepsilon}\left(\lambda-H_{n}\right)_{\alpha j, \gamma j} .
$$

The corresponding canonical distribution of the composite system is

$$
e^{-\beta H_{n}} / \operatorname{Tr} e^{-\beta H_{n}}
$$

and the reduced distribution of the small system is

$$
\frac{\int_{-\infty}^{\infty} e^{-\beta \lambda} \nu^{(n)}(\lambda) d \lambda}{\sum_{\delta= \pm} \int_{-\infty}^{\infty} e^{-\beta \lambda} \nu_{\delta, \delta}^{(n)}(\lambda) d \lambda}
$$

where $(\mathrm{cf}(3.3))$

$$
\nu_{\alpha \gamma}^{(n)}(\lambda)=n^{-1} \sum_{j=1}^{n} \delta\left(\lambda-H_{n}\right)_{\alpha j, \gamma j}
$$

By using standard techniques of random matrix theory (see e.g. [8, 15]), it can be shown that with probability 1 with respect to the distribution (2.3) of the interaction matrix the $n \rightarrow \infty$ limit of (3.3) is

$$
\bar{\nu}_{\alpha \gamma}=\delta_{\alpha, \gamma} \bar{\nu}_{\alpha}, \quad \bar{\nu}_{\alpha}(\lambda)=(2 \varepsilon)^{-1} \int_{\lambda-\varepsilon}^{\lambda+\varepsilon} \nu_{\alpha}(\mu) d \mu
$$

with

$$
\nu_{\alpha}(\lambda)=\pi^{-1} \Im r_{\alpha}(\lambda+i 0) .
$$

The pair $r_{\alpha}(z)$ solves the system of two coupled functional equations

$$
r_{a}(z)=\int_{-\infty}^{\infty} \frac{\nu_{0}(E) d E}{E+s \alpha-z-v^{2} r_{-\alpha}(z)}, \alpha= \pm
$$

with $\nu_{0}$, defined by (2.1) - (2.2). The solution is unique in the class of functions analytic for $\Im z \neq z$, and satisfying the condition $\Im r_{a}(z) \cdot \Im z>0, z \neq 0$.

The $n \rightarrow \infty$ limit of the reduced microcanonical distribution (3.2) is then

$$
\bar{\omega}_{\alpha \gamma}(\lambda)=\delta_{\alpha, \gamma} \bar{\omega}_{\alpha}(\lambda), \quad \bar{\omega}_{\alpha}(\lambda)=\frac{\bar{\nu}_{\alpha}(\lambda)}{\bar{\nu}_{+}(\lambda)+\bar{\nu}_{-}(\lambda)}
$$

Correspondingly, the $n \rightarrow \infty$ limit of the reduced canonical distribution (3.5) is

$$
\rho_{\alpha \gamma}(\beta)=\delta_{a, \gamma} \rho_{\alpha}(\beta), \quad \rho_{\alpha}(\beta)=\frac{\int_{-\infty}^{\infty} e^{-\beta \lambda} \nu_{\alpha}(\lambda) d \lambda}{\sum_{\delta= \pm} \int_{-\infty}^{\infty} e^{-\beta \lambda} \nu_{\delta}(\lambda) d \lambda} .
$$

The absence of off-diagonal elements in $\rho(\beta)$ is due to the special structure of the interaction (proportional to the $\sigma_{x}$ matrix). This is a rather common phenomenon in random 
matrix theory and many ensembles, studied so far, have this property. If, on the other hand, one puts an arbitrary 2 by 2 matrix instead of $\sigma_{x}$ then $\rho(\beta)$ will be non-diagonal.

In the next section we will consider models of $\mathcal{S}_{n}$ in which (3.10) and (3.11) are the same for the proper choice of $\beta$. It will turn out that we will have not only the "equivalence of ensembles" but also that the $\rho_{\alpha}(\beta)$ will be independent of the interactions and therefore be of the Gibbs form,

$$
\frac{e^{-\beta s \alpha}}{2 \cosh \beta s}
$$

\subsection{A model for the reservoir.}

We will consider now a model in which $\mathcal{S}_{n}$ consists of $J$ particles or other subsystems, each having $m$ levels (orbitals). We then have $n=m^{J}$. We assume further that the subsystems do not interact. The (normalized) density of states $\nu_{0}^{(n)}(2.1)$ is then the $J$ fold convolution of the (normalized) density of states $q_{m}$ of a subsystem, and we assume that in the limit $m \rightarrow \infty q_{m}$ will approach some normalized density $q$.

Following the limit $m \rightarrow \infty$ we will take the limit $J \rightarrow \infty$. Note that this is very different from the usual thermodynamic limit, e.g. for $J$ particles on a lattice of $m$ sites, where $J$ would grow with $m$ in such a way that we would have for large $J$ that the number of levels between $E=J e$ and $E=J e+d E$ grows as $\exp \left\{J S_{J}(e)\right\} d E$. The entropy $S_{J}(e)$ would then be a monotone increasing function of $e$ which would approach, as $J \rightarrow \infty$, the entropy/per particle, $S(e)$ with $S^{\prime}(e)=\beta$, the reciprocal temperature of the reservoir. The same happens in spin systems where $m$ remains finite as $J \rightarrow \infty$, in which case $S(e)$ would have a maximum at some finite $e$, after which it would decrease.

The reason we cannot follow this procedure here is the dissonance between random matrix theory and thermodynamic behavior already mentioned above. To get a (normalized) density of states for the thermodynamic system would require that $\exp \left\{J S_{J}(E / J)\right\} / m^{J}$ have a limit as $J \rightarrow \infty$ which is not the case.

We are therefore forced to keep $J$ fixed while taking the limit $m \rightarrow \infty$, yielding a continuous $q$ and $\nu_{0}$ as its $J$-fold convolution. This form of $\nu_{0}$ is standard in probabilities rather than in statistical mechanics. However, they both suggest that we consider cases where

$$
\lim _{J \rightarrow \infty} s_{J}(e)=s(e), \quad s_{J}(e)=J^{-1} \log \nu_{0}(J e) .
$$

The function $s$, appearing above, is in fact the rate function of the large deviation theory (see e.g. [4) and can also be seen as an analog of the entropy of statistical mechanics, although, since $\nu_{0}$ is normalized to unity, the analogy can not be too close. In particular, $s$ will be negative everywhere, since $\nu_{0}(J e) \rightarrow 0$ as $J \rightarrow \infty$, unlike the genuine entropy in quantum statistical mechanics.

Formula (3.13) suggests the following one:

$$
\nu_{0}(J e+\varepsilon) / \nu_{0}(J e)=\exp \left\{J\left[s_{J}(e+\varepsilon / J)-s_{J}(e)\right]\right\} \rightarrow e^{s^{\prime}(e) \varepsilon}, J \rightarrow \infty .
$$

Hence, the equality

$$
s^{\prime}(e)=\beta,
$$

analogous to the well known statistical mechanics relation between the entropy and the temperature, implies the formula

$$
\nu_{0}(J e+\varepsilon) / \nu_{0}(J e) \rightarrow e^{\beta \varepsilon}
$$


To obtain a positive $\beta$ we have to choose an interval on which $s(e)$ of (3.13) increases.

However, for the analysis of (3.10), and (3.11), as well as in the study of the time dependent properties of the model, we will need the asymptotic formula

$$
\nu_{\alpha}(J e) / \nu_{0}(J e) \rightarrow e^{-\beta s \alpha}, J \rightarrow \infty
$$

where $e$ and $\beta$ are related by (3.15). This requires certain conditions on $q$ (in particular, the existence of certain exponential moment) and a certain amount of technicalities. To keep our presentation simple we consider a model case, where $q$ is the Gaussian density

$$
q(e)=\frac{1}{\left(2 \pi a^{2}\right)^{1 / 2}} \exp \left\{-\left(e-e_{0}\right)^{2} / 2 a^{2}\right\}
$$

In this case we have immediately

$$
\nu_{0}(E)=\frac{1}{\left(2 \pi J a^{2}\right)^{1 / 2}} \exp \left\{-\left(E-J e_{0}\right)^{2} / 2 J a^{2}\right\}
$$

and, hence, in view of (3.13),

$$
s(e)=-\left(e-e_{0}\right)^{2} / 2 a^{2} .
$$

Furthermore, we prove in Appendix 1 that if $\nu_{0}$ is given by (3.19) then the density $\nu_{\alpha}$, defined by (3.8) - (3.9), verifies the asymptotic relation (3.17).

It is easy now to show that this model yields a reduced density matrix of $\mathcal{S}_{2}$ that is diagonal in $\alpha$ and has the same Gibbs form (3.12) for both the microcanonical and the canonical cases:

$$
\left.\lim _{J \rightarrow \infty} \lim _{m \rightarrow \infty} \frac{\bar{\nu}_{\alpha}^{(n)}(\lambda)}{\sum_{\gamma= \pm} \bar{\nu}_{\gamma}^{(n)}(\lambda)}\right|_{\lambda=J e}=\lim _{J \rightarrow \infty} \lim _{m \rightarrow \infty} \frac{\int_{-\infty}^{\infty} e^{-\beta J e} \nu_{\alpha}^{(n)}(J e) d e}{\sum_{\gamma= \pm} \int_{-\infty}^{\infty} e^{-\beta J e} \nu_{\gamma}^{(n)}(J e) d e}=\frac{e^{-\beta s \alpha}}{2 \cosh \beta s},
$$

provided that the energy $e$ "per particle" and the temperature $\beta$ of the reservoir are related via the usual equation (3.15). The first formula follows immediately from (3.17). To obtain the second formula we have to take into account that (3.15) is the saddle point condition for the respective integrals in the $n=\infty$ version (3.11) of the canonical reduced density matrix (3.5).

We see that because of the rather "non-macroscopic" (in the thermodynamic sense) nature of the density of states the random matrix model of the reduced equilibrium description does not exhibit, in general, properties that are natural to expect from the statistical mechanics point of view. The same is valid for the time evolution, as will be seen in the next sections. However, the above ansatz for the density of states (be it given by (3.19) or something different) leads to the equivalence of the reduced microcanonical and canonical description in the $J \rightarrow \infty$ limit of our composite system, if we assume, in addition, a natural from the statistical mechanics point of view relation (3.15) between the energy and the temperature.

\section{Time Evolution}

\subsection{General formulas.}


Assume that the energy $E_{k}$ in (2.12) belongs to a subsequence $\left\{E_{k_{n}}\right\}$ that converges to a given energy $E$ of the reservoir such that $\nu_{0}(E)>0$. By using an extension of the techniques, presented in [8, 15], one obtains the following expression for the $n \rightarrow \infty$ limit of the transfer matrix (2.12):

$$
\begin{aligned}
T_{\alpha \beta \gamma \delta}(E, t) & = \\
& =\frac{1}{(2 \pi i)^{2}} \int_{C} \int_{C} d z_{1} d z_{2} e^{i t\left(z_{1}-z_{2}\right)} r_{\alpha \beta \gamma \delta}\left(E, z_{1}, z_{2}\right),
\end{aligned}
$$

where the contour $C$ encircles the real axis,

$$
r_{\alpha \beta \gamma \delta}\left(E, z_{1}, z_{2}\right)=\lim _{n \rightarrow \infty}\left\langle\sum_{j=1}^{n} R_{\gamma k, \delta j}\left(z_{1}\right) R_{\alpha j, \beta k}\left(z_{2}\right)\right\rangle,
$$

and $R(z)=\left(H_{n}-z\right)^{-1}$ is the resolvent of the Hamiltonian (2.5). The "two-point" functions $r_{\alpha \beta \gamma \delta}\left(E, z_{1}, z_{2}\right)$ are analytic in $z_{1}$ and in $z_{2}$ outside the real axis and have the form

$$
\begin{aligned}
r_{\alpha \beta \gamma \delta}\left(E, z_{1}, z_{2}\right) & =r_{\gamma}\left(E, z_{1}\right) r_{\beta}\left(E, z_{2}\right) D_{\gamma, \beta}^{-1}\left(z_{1}, z_{2}\right) \\
& \times\left(\delta_{\alpha, \beta} \delta_{\gamma, \delta}+v^{2} \delta_{-\alpha, \beta} \delta_{-\gamma, \delta} r_{-\gamma,-\beta}\left(z_{1}, z_{2}\right)\right)
\end{aligned}
$$

Here the "one-point" functions $r_{\alpha}(E, z), \alpha= \pm$ are

$$
r_{a}(E, z)=\frac{1}{E+s \gamma-z-v^{2} r_{-\alpha}(z)}
$$

$r_{\alpha}(z), \alpha= \pm$ solve system (3.9),

$$
D_{\gamma, \beta}\left(z_{1}, z_{2}\right)=1-v^{4} r_{\gamma, \beta}\left(z_{1}, z_{2}\right) r_{-\gamma,-\beta}\left(z_{1}, z_{2}\right),
$$

and

$$
r_{\gamma, \beta}\left(z_{1,} z_{2}\right)=\int r_{\gamma}\left(E, z_{1}\right) r_{\beta}\left(E, z_{2}\right) \nu_{0}(E) d E
$$

is another two-point function.

These formulas, and (2.11) lead to the following expression for the $n \rightarrow \infty$ limit of the reduced density matrix

$$
\begin{aligned}
\rho_{\alpha, \delta}(E, t) & =\frac{1}{(2 \pi i)^{2}} \int_{C} d z_{2} \int_{C} d z_{1} e^{i t\left(z_{1}-z_{2}\right)} \\
& \times \frac{r_{\alpha}\left(E, z_{1}\right) r_{\delta}\left(E, z_{2}\right) \rho_{\alpha, \delta}(0)+v^{2} r_{-\alpha}\left(E, z_{1}\right) r_{-\delta}\left(E, z_{2}\right) r_{\alpha, \delta}\left(z_{1}, z_{2}\right) \rho_{-\alpha,-\delta}(0)}{1-v^{4} r_{\alpha, \delta}\left(z_{1}, z_{2}\right) r_{-\alpha,-\delta}\left(z_{1}, z_{2}\right)} .
\end{aligned}
$$

Hence the limiting transition probabilities, corresponding to $\rho_{\alpha, \alpha}(0)=\delta_{\alpha \beta}$, are

$$
p_{\alpha, \beta}(E, t)=\rho_{\alpha, \alpha}(E, t) \text {. }
$$

4.2. Reduced density matrix and the transition probabilities in the infinite time limit. 
By using the analyticity of the integrand of (4.7) in $z_{1}$ and in $z_{2}$, we can write the following integral representation for the diagonal entries of the reduced density matrix of the system

$$
\begin{aligned}
\rho_{\alpha, \alpha}(E, t) & =-\frac{1}{(2 \pi i)^{2}} \int_{-\infty}^{\infty} d \lambda_{2} \int_{-\infty}^{\infty} d \lambda_{1} e^{i t \delta \lambda} \\
& \times \frac{\delta r_{\alpha}(E)\left(\delta \lambda+v^{2} \delta r_{\alpha}\right) \rho_{\alpha, \alpha}(0)+v^{2} \delta r_{-\alpha}(E) \delta r_{\alpha} \rho_{-\alpha,-\alpha}(0)}{\delta \lambda\left(\delta \lambda+v^{2} \delta r_{\alpha}+v^{2} \delta r_{-\alpha}\right)}
\end{aligned}
$$

Here the integration path in $\lambda_{1}$ encircles $\lambda_{2}$ from below in the clockwise direction, $\delta \lambda=$ $\lambda_{1}-\lambda_{2}$

$$
\begin{aligned}
\delta r_{\alpha}(E) & =r_{\alpha}\left(E, \lambda_{1}-i 0\right)-r_{\alpha}\left(E, \lambda_{2}+i 0\right), \\
\delta r_{\alpha} & =r_{\alpha}\left(\lambda_{1}-i 0\right)-r_{\alpha}\left(\lambda_{2}+i 0\right),
\end{aligned}
$$

and $r_{\alpha}(z)$ and $r_{a}(E, z)$ are defined in (3.9) and in (4.4).

These formulas imply that the diagonal entries of the reduced density matrix are, in the limit $t \rightarrow \infty$, given by

$$
\rho_{\alpha, \alpha}(E, \infty)=\int_{-\infty}^{\infty} \omega_{\alpha}(\lambda)\left(\sum_{\gamma= \pm} \nu_{\gamma}(E, \lambda) \rho_{\gamma, \gamma}(0)\right) d \lambda
$$

where

$$
\omega_{\alpha}(\lambda)=\frac{\nu_{\alpha}(\lambda)}{\nu_{+}(\lambda)+\nu_{-}(\lambda)}, \alpha= \pm
$$

is the $\varepsilon \rightarrow 0$ form of microcanonical distribution of the small system, defined in (3.10) with $\bar{\nu}_{\alpha}(\lambda)$ of (3.7), replaced by $\nu_{\alpha}(\lambda)$ of (3.8) - (3.9), and

$$
\begin{aligned}
\nu_{\alpha}(E, \lambda) & =\frac{1}{\pi} \Im r_{\alpha}(E, \lambda+i 0) \\
& =\frac{v^{2} \nu_{-\alpha}(\lambda)}{\left(E+s \alpha-\lambda-v^{2} \Re r_{-\alpha}(\lambda+i 0)\right)^{2}+\pi^{2} v^{4} \nu_{-\alpha}^{2}(\lambda)}, \alpha= \pm,
\end{aligned}
$$

where the last equality follows from (4.4). The functions $\nu_{\alpha}(E, \lambda)$ are non-negative and satisfy the relations

$$
\int_{-\infty}^{\infty} \nu_{\alpha}(E, \lambda) d \lambda=\int_{-\infty}^{\infty} \nu_{\alpha}(E, \lambda) d E=1, \int_{-\infty}^{\infty} \nu_{\alpha}(E, \lambda) \nu_{0}(E) d E=\nu_{\alpha}(\lambda), \alpha= \pm .
$$

Relations (4.8) and (4.11) imply that the transition probabilities are given by

$$
p_{\alpha, \gamma}(E, \infty)=\int_{-\infty}^{\infty} \omega_{\alpha}(\lambda) \nu_{\gamma}(E, \lambda) d \lambda
$$

where $\omega_{\alpha}$ is the microcanonical equilibrium distribution (4.12), and $\nu_{\alpha}(E, \lambda)$ is defined in (4.13) - (4.14). This expression satisfies the normalization condition (2.14).

As already remarked at the end of Section 3.1, there is no reason to expect that $\rho(E, \infty)$ will correspond to any equilibrium state for a general $\nu_{0}$. In fact we can show (see Section 7.1 ) that the diagonal entries $\rho_{\alpha, \alpha}(E, \infty)$ of (4.11) will in general depend on $\rho(0)$. The situation is different for the model of the reservoir, introduced in Section 3.2. Indeed, setting $E=J e(\beta)$ in (4.11), where $e(\beta)$ is defined by (3.15), and (3.20), 
we can change to the variable $\lambda=J e$, and replace asymptotically the density $\nu_{\alpha}(J e)$ by $\nu_{0}(J e) \exp \left\{\left(e-e_{0}\right) s \alpha / a^{2}\right\}$, and the density $\nu_{\alpha}(J e(\beta), J e) J$ by $\delta\left(e+a^{2} \beta-e_{0}\right)$ for $J \rightarrow \infty$, according to relations (A.9), and (A.10). As a result we obtain, in the infinite time limit, the canonical Gibbs distribution form (3.21) of the diagonal entries of the mean reduced density matrix of the small system with a temperature, determined by the energy by $\mathcal{S}_{n}$. Note that the interaction does not appear in the limit although it ensures the loss of memory of $\rho(0)$.

\section{$5 \quad$ Van-Hove Limit}

In this limit the coupling constant $v$ of the system-reservoir interaction tends to zero, the time $t$ tend to infinity while the transition rate, given by first order perturbation in the interaction, is kept fixed. In terms of (4.1) this corresponds to letting

$$
v \rightarrow 0, \quad t \rightarrow \infty, \quad \tau=t v^{2} \quad \text { fixed }
$$

after the limit $n \rightarrow \infty$.

By using general formulas (4.7) - (4.3), it is easy to show that the off-diagonal entry $\rho_{+-}$of the reduced density matrix vanishes in the van Hove limit.

Let us consider the diagonal entries $\rho_{\alpha, \alpha}(E, t)$ in this limit. Changing variables to $\lambda_{2}=\lambda, \delta \lambda=v^{2} \xi$ in the integral representation (4.9), and using (4.10), (3.8), and (4.13), we obtain

$$
\left.\delta r_{\alpha}(E)\right|_{\lambda_{1}=\lambda_{2}=\lambda}=-2 \pi i \nu_{\alpha}(E, \lambda),\left.\delta r_{\alpha}\right|_{\lambda_{1}=\lambda_{2}=\lambda}=-2 \pi i \nu_{\alpha}(\lambda)
$$

where now

$$
\nu_{\alpha}(\lambda)=\nu_{0}(\lambda-s \alpha), \nu_{\alpha}(E, \lambda)=\delta(E+s \alpha-\lambda) .
$$

The limit $\rho_{\alpha, \alpha}^{v H}(E, \tau)$ of the diagonal entries, is then given by

$$
\begin{aligned}
\rho_{\alpha, \alpha}^{v H}(E, \tau) & =\frac{1}{2 \pi i} \int_{-\infty}^{\infty} d \lambda \int_{-\infty}^{\infty} d \xi e^{i \tau \xi} \\
& \times \frac{\nu_{\alpha}(E, \lambda)\left[\xi-2 \pi i \nu_{\alpha}(\lambda)\right] \rho_{\alpha, \alpha}(0)-2 \pi i \nu_{-\alpha}(E, \lambda) \nu_{\alpha}(\lambda) \rho_{-\alpha,-\alpha}(0)}{\xi(\xi-i \Gamma(\lambda))}
\end{aligned}
$$

where

$$
\Gamma(\lambda)=2 \pi\left(\nu_{+}(\lambda)+\nu_{-}(\lambda)\right),
$$

and the integration with respect to $\xi$ is taken by encircling the origin from below in the clockwise direction. Computing the integral with respect to $\xi$ by residues, we get (cf Appendix 2)

$$
\begin{aligned}
\rho_{\alpha, \alpha}^{v H}(E, \tau) & =\int_{-\infty}^{\infty}\left(\omega_{\alpha}(\lambda) \sum_{\gamma} \nu_{\gamma}(E, \lambda) \rho_{\gamma, \gamma}(0)\right. \\
& \left.+\frac{\nu_{\alpha}(E, \lambda) \nu_{-\alpha}(\lambda) \rho_{\alpha, \alpha}(0)-\nu_{-\alpha}(E, \lambda) \nu_{\alpha}(\lambda) \rho_{-\alpha,-\alpha}(0)}{\nu_{+}(\lambda)+\nu_{-}(\lambda)} e^{-\tau \Gamma(\lambda)}\right) d \lambda .
\end{aligned}
$$

We see that the time independent part of the formula coincides with the r.h.s. of (4.11), modulo the replacement (5.3). 
Now, taking into account (5.3), we obtain finally that the reduced density matrix in the van Hove limit is

$$
\rho_{\alpha, \gamma}^{v H}(E, \tau)=\delta_{\alpha, \gamma} \rho_{\alpha, \alpha}^{v H}(E, \tau)
$$

where

$$
\begin{gathered}
\rho_{\alpha, \alpha}^{v H}(E, \tau)=\frac{\nu_{0}(E)}{\nu_{0}(E)+\nu_{0}(E+2 s \alpha)} \rho_{\alpha, \alpha}(0)+\frac{\nu_{0}(E-2 s \alpha)}{\nu_{0}(E-2 s \alpha)+\nu_{0}(E)} \rho_{-\alpha,-\alpha}(0) \\
+\frac{\nu_{0}(E+2 s \alpha)}{\nu_{0}(E)+\nu_{0}(E+2 s \alpha)} \rho_{\alpha, \alpha}(0) e^{-\tau \Gamma_{\alpha}(E)}-\frac{\nu_{0}(E-2 s \alpha)}{\nu_{0}(E-2 s \alpha)+\nu_{0}(E)} \rho_{-\alpha,-\alpha}(0) e^{-\tau \Gamma_{-\alpha}(E)},
\end{gathered}
$$

and

$$
\Gamma_{\alpha}(E)=\Gamma(E+s \alpha)=2 \pi\left(\nu_{0}(E)+\nu_{0}(E+2 s \alpha)\right),
$$

with $\Gamma(\lambda)$, given by (15.4). In a general case of an arbitrary density of states $\nu_{0}$ of the reservoir the infinite time part of the reduced density matrix (5.7) in the van Hove limit depends on the initial density matrix $\rho(0)$ of the system, like it was for our general formula (4.11) (see, however, Section 7.2). Besides, the r.h.s. of formula (5.7) contains three "modes": the time independent term, and the two terms with different exponentials in $t$. This implies that the pair $\left(\rho_{++}, \rho_{--}\right)$can not obey a system of two differential equations of the first order, an expected form of the master equation for $\rho_{\alpha, \alpha}^{v H}(E, \tau)$

In fact, it is easy to see that if the first line of (5.7) is independent of $\rho(0)$ for all values of $E$ (recall that this is a free parameter of our model), then

$$
\nu_{0}(E)=C e^{\beta E}
$$

where $C$ and $\beta$ are constants. This form of $\nu_{0}$ leads evidently to the Gibbs form of the equilibrium distributions (3.10), and to the right side of (5.7) having the form

$$
\frac{e^{-\beta s \alpha}}{2 \cosh \beta s}+\frac{e^{-\beta s \alpha}}{2 \cosh \beta s} \rho_{\alpha, \alpha}(0) e^{-\tau \Gamma_{\alpha}(E)}-\frac{e^{\beta s \alpha}}{2 \cosh \beta s} \rho_{-\alpha,-\alpha}(0) e^{-\tau \Gamma_{-\alpha}(E)}
$$

with

$$
\Gamma_{\alpha}(E)=4 \pi \nu_{0}(E+s \alpha) \cosh \beta s .
$$

We note, however, that while the form (5.9) of $\nu_{0}$ for all $E$ is incompatible with the normalization condition (2.2), it can be valid "locally" in $E$ as in formula (3.16). We have seen in Section 3.2 that (3.16) implies the Gibbs form of the equilibrium densities matrices (3.10), and (3.11) in the zero coupling limit.

Likewise, by using the asymptotic relation (3.16) in the time-independent term of (5.7), we obtain the first term of (5.10) i.e. the Gibbs form of the time-independent term (5.7), after the replacement $E$ by $J e(\beta)$ and the subsequent limit $J \rightarrow \infty$.

The situation with the time dependent part of the reduced density matrix in the considered regime (5.1)-3.14) is less satisfactory. Indeed, according to relation (3.19), the density of states $\nu_{0}(J e(\beta))$ is exponentially small for large $J$ (except the case, where $e(\beta)-e_{0}$ is of the order $J^{-1 / 2}$, that corresponds to the "infinite temperature" $\beta=0$ ). Hence the decay rate (5.11) $\Gamma$ will also be exponentially small in $J$.

A simple way to make $\Gamma_{\alpha}(E)$ in (5.8) non-vanishing as $J \rightarrow \infty$ is to assume that the coupling constant $v$ in (2.5) depends on $J$. This can be done if we write $v$ as the product 
of two factors $v_{1}$ and $v_{2}$, use $v_{1}$ in the definition (5.1) of the van Hove limit and keep $v_{2}$ in the decay rate. This yields

$$
\Gamma_{\alpha}(E)=4 \pi v_{2}^{2}\left(\nu_{0}(E)+\nu_{0}(E+s \alpha)\right)
$$

instead of (5.8) and allows us to rescale the decay rate by setting $v_{2}$ proportional to $\left(\nu_{0}(E)+\nu_{0}(E+s \alpha)\right)^{-1}$. Since the parameter $E$ appears in the initial formulas (2.13) and (2.12) via the condition $E_{k}=E$, we see that we have to make the interaction term in (2.5) dependent on the energy levels of the reservoir. In the next section we consider a version of the model, possessing this property.

\section{A More General Interaction Model}

In this section we consider a model, given by the Hamiltonian (2.5), in which the system - reservoir interaction $w_{n}$ is a real symmetric Gaussian random matrix with statistically dependent entries whose covariances are given by the relations (cf (2.3) $)$ :

$$
\mathbf{E}\left\{w_{j k}\right\}=0, \quad \mathbf{E}\left\{w_{j k} w_{l m}\right\}=f\left(E_{j}, E_{k}\right)\left(\delta_{j l} \delta_{k m}+\delta_{j m} \delta_{k l}\right), j, k=1, \ldots, n,
$$

where $f\left(E, E^{\prime}\right)$ is a non-negative symmetric function. Assuming that $f\left(E, E^{\prime}\right)$ vanishes if $\left|E-E^{\prime}\right|>b$, for some $b$, we obtain the so-called band matrices, whose entries are non zero inside the "band" of the width $b$. The case $f\left(E, E^{\prime}\right) \equiv 1$ corresponds to (2.3). Similar models were considered in [14, 16, 2, 12].

A somewhat tedious calculation leads to a transfer matrix $T_{\alpha \beta \gamma \delta}(E, t)$ that is given again by formula (4.1) in which now (cf (4.3) $)$

$$
\begin{aligned}
r_{\alpha \beta \gamma \delta}\left(E, z_{1}, z_{2}\right) & =r_{\gamma}\left(E, z_{1}\right) r_{\beta}\left(E, z_{2}\right) \\
& \times\left(1-S_{-\alpha,-\delta}\left(z_{1}, z_{2}\right) S_{\alpha, \delta}\left(z_{1}, z_{2}\right)\right)^{-1} \\
& \times\left(\delta_{\alpha, \beta} \delta_{\gamma, \delta}+\delta_{-\alpha, \beta} \delta_{-\gamma, \delta} s_{-\gamma,-\beta}\left(z_{1}, z_{2}\right)\right)
\end{aligned}
$$

where $(\operatorname{cf}(4.4))$

$$
r_{\alpha}(E, z)=\frac{1}{E+s \alpha-z-\Delta_{-\alpha}(E, z)} .
$$

The "self-energies" $\Delta_{\alpha}(E, z), \alpha= \pm$ solve the system of two nonlinear integral equations $(\operatorname{cf}(3.9))$

$$
\Delta_{\alpha}(E, z)=\int_{-\infty}^{\infty} \frac{v^{2} f\left(E, E^{\prime}\right) \nu_{0}\left(E^{\prime}\right) d E^{\prime}}{E^{\prime}+s \alpha-z-\Delta_{-\alpha}\left(E^{\prime}, z\right)}, \alpha= \pm,
$$

$S_{\alpha, \delta}\left(z_{1}, z_{2}\right)$ is an integral operator, acting on a function $\phi$ of $E$ by the formula

$$
\left(S_{\alpha, \delta}\left(z_{1}, z_{2}\right) \phi\right)(E)=\int_{-\infty}^{\infty} v^{2} f\left(E, E^{\prime}\right) r_{\alpha}\left(E^{\prime}, z_{1}\right) r_{\delta}\left(E^{\prime}, z_{2}\right) \phi\left(E^{\prime}\right) \nu_{0}\left(E^{\prime}\right) d E^{\prime}
$$

and

$$
s_{\alpha, \delta}\left(z_{1}, z_{2}\right)=\left(S_{\alpha, \delta}\left(z_{1}, z_{2}\right) \mathbf{1}\right)(E) .
$$

It is easy to see that if $f\left(E, E^{\prime}\right) \equiv 1$, then the above formulas coincide with our previous formulas (4.3) - (4.6) in which $\Delta_{\alpha}(E, z)=v^{2} r_{\alpha}(z)$. 
We show in Section 7.3 that particular asymptotic cases of the above formulas correspond to the results of papers [14, 16].

Consider now the van-Hove limit of this model as defined in (5.1). One obtains then the expressions (5.7) - (5.8) in which $\nu_{\alpha}(\lambda)=\nu_{0}(\lambda-s \alpha)$ is replaced by

$$
\nu_{0}(\lambda-s \alpha) f(\lambda+s \alpha, \lambda-s \alpha)
$$

Following [12, we choose the form-factor $f$ in (6.1) in the form

$$
f(\lambda, \mu)=\frac{w(\lambda-\mu)}{\sqrt{\nu_{0}(\lambda) \nu \nu_{0}(\lambda)}}, w(\lambda)=w(-\lambda),
$$

which is in agreement with our discussion at the end of the previous section. This leads to the following form of the diagonal entries of the reduced density matrix (cf (5.10) $)$ :

$$
\rho_{\alpha, \alpha}(E, \tau)=\frac{e^{-\beta s \alpha}}{2 \cosh \beta s}+\frac{e^{\beta s \alpha} \rho_{\alpha, \alpha}(0)-e^{-\beta s \alpha} \rho_{-\alpha,-\alpha}(0)}{2 \cosh \beta s} e^{-\tau \Gamma},
$$

where now (cf (5.11) $)$

$$
\Gamma=4 \pi w(2 s) \cosh \beta s .
$$

Unlike (5.7), obtained in Section 5 for $f \equiv 1$, the r.h.s. of (6.9) contains only two "modes", the time independent term and the term with the exponential $e^{-\tau \Gamma}$. As a result, $\rho_{\alpha, \alpha}(E, \tau), \alpha= \pm$ is the solution of the system of two differential equations:

$$
\frac{d}{d t} \rho_{\alpha, \alpha}=-2 \pi w(2 s)\left(e^{\beta s \alpha} \rho_{\alpha, \alpha}-e^{-\beta s \alpha} \rho_{-\alpha,-\alpha}\right)
$$

Eq. (6.11) is a version of the master equation which is believed to describe, on an appropriate time scale [7, 9, 17, the approach to equilibrium of a small system interacting weakly with a reservoir. An analog of (6.11) was obtained in 12] (see Section 6, in particular formulas (52) - (53) there). From the probabilistic point of view (6.11) describes the two-state Markov process, known as the random telegraph signal or the dichotomy process (see e.g. [10]); see discussion after (7.14).

\section{Some Properties of the Models}

In this section we discuss certain, mostly asymptotic, properties of the models, specified in Sections 2 and 6, that do not require the special structure of the reservoir, introduced in Section 3.2.

\subsection{The dependence of the reduced density matrix on the initial density matrix.}

We consider here the dependence of the $t \rightarrow \infty$ limit (4.11) of the diagonal entries of the reduced density matrix on the initial density matrix $\rho(0)$ of the small system in the model of Section 2.

It is easy to find from (4.11) that if the $\rho_{\alpha, \alpha}(E, \infty)$ are independent of $\rho(0)$, then they have the form

$$
\rho_{\alpha, \alpha}(E, \infty)=\frac{1}{2} \int_{-\infty}^{\infty} \frac{\nu_{+}(E, \lambda)+\nu_{-}(E, \lambda)}{\nu_{+}(\lambda)+\nu_{-}(\lambda)} \nu_{\alpha}(\lambda) d \lambda
$$


corresponding to $\rho_{ \pm}(0)=1 / 2$ in (4.11), and that if the $\rho_{\alpha, \alpha}(E, \infty)$ are independent of $\rho(0)$ for all $E$, then

$$
\int_{-\infty}^{\infty} \frac{\left(\nu_{+}(\lambda)-\nu_{-}(\lambda)\right)^{2}}{\nu_{+}(\lambda)+\nu_{-}(\lambda)} d \lambda=0 .
$$

Thus, the equality $\nu_{+}(\lambda)=\nu_{-}(\lambda)$ has to be valid for all $\lambda$ which is impossible for the Hamiltonian (2.5) with $s \neq 0$, i.e. if the small system has a nontrivial dynamics. Hence, there will in general exist energies of the reservoir for which the $t=\infty$ reduced density matrix of the model of Section 2 depends on $\rho(0)$.

\subsection{The regime of an almost flat density of states of the reservoir.}

There is a case where the $\rho_{\alpha, \alpha}(E, \infty)$ are equal to $1 / 2$ and the off diagonal entries vanish: thus corresponding in some way to the Gibbs distribution (3.21) at infinite temperature $\beta=0$. This occurs for an "almost flat" density of states $\nu_{0}$ of the reservoir. More precisely, we assume that $\nu_{0}$ has the form (cf (3.19) $)$

$$
\nu_{0}(\lambda)=\frac{1}{a} \varphi\left(\frac{\lambda}{a}\right),
$$

where $\varphi$ is a non-negative function, having unit integral, and $a$ is the biggest parameter having the dimension of energy of the problem. We assume in addition that the parameter

$$
\frac{\pi \varphi(0) v^{2}}{a}=\pi \nu_{0}(0) v^{2}:=A
$$

is fixed, i.e. that the coupling constant is of the order $\sqrt{a}$. Hence, the regime corresponds formally to the following limiting procedure:

$$
a \rightarrow \infty, v \rightarrow \infty, A=\pi \varphi(0) v^{2} / a \text { is fixed. }
$$

By using the relation

$$
\int_{-\infty}^{\infty} \frac{d x}{x-z}=\pi i \sigma(z), \sigma(z)=\operatorname{sign}(\Im z)
$$

we obtain that in this regime

$$
v^{2} r_{\alpha}(z)=A i \sigma(z), r_{\alpha}(E, z)=\frac{1}{E+s \alpha-z-A i \sigma(z)}
$$

Thus, according to (3.8) and (4.13), we have (cf (4.13) $)$

$$
\nu_{\alpha}(E, \lambda)=\frac{A}{\pi\left[(E+s \alpha-\lambda)^{2}+A^{2}\right]}, \quad v^{2} \nu_{\alpha}(\lambda)=A / \pi .
$$

Now, computing respective integrals in (4.9) (see Appendix 2), we find that

$$
\rho_{\alpha, \alpha}(E, t)=\frac{1}{2}+\alpha \frac{\rho_{+,+}(0)-\rho_{-,-}(0)}{2} e^{-\Gamma t},
$$

where

$$
\Gamma=4 A=4 \pi v^{2} \nu_{0}(0)
$$


Formula (17.10) for the rate of the exponentially fast convergence to the infinite time (equilibrium) limit (17.11) is an analog of the Fermi Golden Rule in our context.

These formulas can be obtained as a particular case of (5.7), and (5.8), corresponding to the replacement of all arguments in $\nu_{0}$ by zeros, which is natural in view of (17.3) and the spirit of the approximation, according to which $a$ is the biggest parameters with the dimension of energy, i.e., $a>>E, s \alpha$, hence $\nu_{0}(E \pm 2 s \alpha) \simeq \nu_{0}(0)$. Note, however, that (5.7), and (5.8) correspond to the small coupling case (5.1), while (7.9), and (7.10) are obtained assuming, at least formally, that $v \rightarrow \infty$ (see (7.5)) It can also be shown that in this asymptotic regime the off-diagonal entry $\rho_{+,-}(E, t)$ of the reduced density matrix decay exponentially fast as $t \rightarrow \infty$ with the rate $2\left(A-\sqrt{A^{2}-s^{2}}\right)$, if $0 \leq s \leq A$, and with the rate $2 A$, if $s \geq A$.

It follows then from (17.9) that in this approximation the $t \rightarrow \infty$ limit of the reduced density matrix is

$$
\rho(E, \infty)=\left(\begin{array}{cc}
1 / 2 & 0 \\
0 & 1 / 2
\end{array}\right)
$$

i.e. it has the "infinite temperature" $(\rho(0)$-independent) Gibbs form of (3.21).

Likewise, we have for the transition probabilities from (4.8), and (7.9):

$$
p_{\alpha, \delta}(E, t)=\frac{1}{2}+\frac{\alpha \delta}{2} e^{-\Gamma t}
$$

in particular,

$$
\rho(E, \infty)=\left(\begin{array}{ll}
1 / 2 & 1 / 2 \\
1 / 2 & 1 / 2
\end{array}\right) .
$$

Formula (7.9) is derived in Appendix 2. The derivation makes explicit a mathematical mechanism of the exponential decay of the second term (exponentially fast convergence to equilibrium). Namely, to compute the double integral in (4.11) with the functions $r_{\alpha}(z)$ and $r_{\alpha}(E, z)$, given by (7.7), we make analytic continuation of $r_{\alpha}\left(\lambda_{1}-i 0\right)$ to the upper half-plane and of $r_{\alpha}\left(\lambda_{2}+i 0\right)$ to the lower half-plane, which are the "non-physical" sheets for these functions. This procedure can be viewed as a simple version of analytic continuation of the resolvent of a self-adjoint operator via the cut, determined by its spectrum, i.e. as a "toy" case of analysis of resonances. In the recent rigorous studies [1, 6] of the return to equilibrium this analysis was carried out by using the techniques of the complex dilatation, applied to the Liouville operator of the composite system.

Formula (17.9) implies that the $\rho_{\alpha, \alpha}(E, t)$ satisfy the system of two differential equations

$$
\frac{d}{d t} \rho_{\alpha, \alpha}=-\frac{\alpha \Gamma}{2}\left(\rho_{\alpha, \alpha}-\rho_{-\alpha,-\alpha}\right), \alpha= \pm
$$

Eq. (7.14) describes a two-state Markov process, known as the random telegraph signal or the dichotomy process (see e.g. [10]). The process assumes alternatively two values on intervals, whose lengths are independent identically distributed random variables with the probability density $\Gamma e^{-\Gamma \tau}$. The equation plays the role of a master equation for our model in the asymptotic regime (7.3) - (17.4) of the almost flat density of states of the reservoir. It is the $\beta=0$ version of the equation (6.11).

\subsection{Special cases of the generalized model.}


We consider here certain asymptotic cases of the model of Section 6, defined by (6.1). Namely, we assume that the density of states $\nu_{0}$ of the reservoir has the "almost flat" form (17.3) - (7.4), that the form-factor $f\left(E, E^{\prime}\right)$ of (6.1) is

$$
f\left(E, E^{\prime}\right)=w\left(\frac{E-E^{\prime}}{b}\right)
$$

and that, in addition to (17.4), the inequality

$$
a \gg b
$$

is valid. This leads to the following system of equations for the self-energies $\Delta_{\alpha}$ of (6.4):

$$
\Delta_{\alpha}(E, z)=A \int_{-\infty}^{\infty} \frac{w(x / b) d x}{x+E+s \alpha-z-\Delta_{-\alpha}(E, z)},
$$

where $A$ is defined in (7.4). To compute the integral we take the convenient "Lorenzian" form of $w$

$$
w(x)=\frac{1}{1+x^{2}},
$$

and we obtain

$$
\Delta_{\alpha}(E, z)=\frac{\pi A b}{E+s \alpha-z-\Delta_{-\alpha}(E, z)-i b \sigma(z)},
$$

where $\sigma(z)$ is defined in (7.6). Similar argument, applied to the operator $S_{\alpha, \delta}$ of (6.5), shows that (6.2) has the form (4.3) in which $v^{2} r_{\alpha}$ is replaced by $\Delta_{\alpha}$ from (17.19).

Before analyzing the reduced density matrix in this asymptotic regime, consider the case, where $b \gg s$, i.e. where the system dynamics is negligible. In this case the r.h.s of (7.19) coincides with the r.h.s. of (7.7), thus the diagonal entries of the reduced density matrix are given by (7.9). This result for the transition probability was obtained in [14] by using the Dyson series in powers of the system-reservoir interaction for the evolution operator $\exp \left\{-\left.i t H_{n}\right|_{s=0}\right\}$ and keeping only those terms of the respective series that are relevant in the $n \rightarrow \infty$ limit. They considered the regime defined by (17.3), (17.4), and used the inequalities $a \gg b \gg s$.

On the other hand, in the regime (7.16) but with the energy $s$ of the small system being of the same order as the range of energies $b$, participating in the system-reservoir interaction, we obtain from (17.19)

$$
\Delta_{\alpha}(E, z)=\frac{\pi A b}{s \alpha-i b \sigma(z)}
$$

By using (7.20) and the above remark on the form of (6.2), we obtain again formula (7.9) in which $\Gamma$ of (7.10) is replaced by

$$
\Gamma_{1}=\Gamma \frac{b^{2}}{s^{2}+b^{2}}
$$

This result was obtained in [16], by using a more sophisticated version of the perturbation theory, proposed in [14. 


\section{Appendices}

\section{Large energy tails of $\nu_{\alpha}(\lambda)$}

We will prove here the asymptotic formula (A.9). Recall that the density $\nu_{\alpha}$ is defined via its Stieltjes transform

$$
r_{\alpha}(z)=\int \frac{\nu_{\alpha}(\lambda) d \lambda}{\lambda-z}, \Im z \neq 0
$$

by formula (3.8), and by the system (3.9). Because of formula (3.8) it suffices to consider the case, where $\Im z \geq 0$. By using the identity

$$
\frac{1}{\lambda-\zeta}=i \int_{0}^{\infty} e^{-i(\lambda-\zeta) t} d t, \Im z>0,
$$

and taking into account that, according to A.1 $, \Im r_{\alpha}(z) \cdot \Im z>0, \Im z \neq 0$, we can write (3.9) for $\Im z>0$ as

$$
r_{\alpha}(z)=i \int_{0}^{\infty} e^{-i t\left(s \alpha-z-v^{2} r_{-\alpha}(z)\right)} f_{J}(t) d t
$$

where

$$
f_{J}(z)=\int_{-\infty}^{\infty} e^{-i t E} \nu_{0}(E) d E=\exp \left\{-i t e_{0} J-\frac{J t^{2} a^{2}}{2}\right\} .
$$

In writing the last equality we used the form (3.19) of the density of states of the reservoir. Since $\Im r_{\alpha}(E+i 0)$ is non-negative for $\Im z \geq 0$, formulas (A.2) and (A.3) imply the inequality

$$
\left|r_{\alpha}(E+i 0)\right| \leq\left(\pi / 2 J a^{2}\right)^{1 / 2}
$$

The same formulas lead to the relation

$$
r_{\alpha}(J e+i 0)=i e^{\frac{J^{2} \xi^{2} a^{2}}{2}} \int_{0}^{\infty} e^{-\frac{J a^{2}}{2}(t-i \xi)^{2}-i t\left(s \alpha-v^{2} r_{-\alpha}(J e+i 0)\right)} d t
$$

where

$$
\xi=x / a^{2}, x=e-e_{0} .
$$

Replace the integral in the r.h.s. of (A.5) by the sum of the two integrals $I_{1}$ and $I_{2}$ over the intervals $[0, i \xi]$ and $[i \xi, \infty+\xi y+)$ respectively. The first integral can be written as

$$
I_{1}(e)=-\xi \int_{0}^{1} e^{-\frac{J \xi^{2} a^{2}}{2} u+\xi g_{1}(u)\left(s \alpha-v^{2} r_{-\alpha}(J e+i 0)\right)} g_{2}(u) d u
$$

where

$$
g_{1}(u)=\frac{u}{1+\sqrt{1-u}}, g_{2}(u)=\frac{1}{2 \sqrt{1-u}} .
$$

In view of (A.4) the leading contribution to $I_{1}$ as $J \rightarrow \infty$ is due to the lower integration limit $u=0$. This yields the asymptotic formula

$$
I_{1}(e)=-\frac{1}{J x}-\frac{1+\xi\left(s \alpha-v^{2} r_{-\alpha}(e+i 0)\right)}{J^{2} a^{4} \xi^{3}}+O\left(J^{-3}\right), J \rightarrow \infty
$$

where $x$ and $\xi$ are defined in (A.6). 
The integral $I_{2}$ can be transformed to the form

$$
I_{2}(e)=i e^{-\frac{J \xi^{2} a^{2}}{2}} \int_{0}^{\infty} e^{-\frac{J u^{2} a^{2}}{2}+(\xi-i u)\left(s \alpha-v^{2} r_{-\alpha}(J e+i 0)\right)} d u .
$$

The leading contribution to $I_{2}$ is also due to the lower integration limit $u=0$, hence we have

$$
\begin{aligned}
I_{2}(e) & =i e^{-\frac{J \xi^{2} a^{2}}{2}} e^{-\xi\left(s \alpha-v^{2} r_{-\alpha}(J e+i 0)\right)} \int_{0}^{\infty} e^{-\frac{J u^{2} a^{2}}{2}} d u\left(1+O\left(J^{-1}\right)\right) \\
& =i e^{-\frac{J \xi^{2} a^{2}}{2}} e^{-\xi\left(s \alpha-v^{2} r_{-\alpha}(J e+i 0)\right)} \sqrt{\frac{\pi}{2 J a^{2}}}\left(1+O\left(J^{-1}\right) .\right.
\end{aligned}
$$

These formulas imply (cf (A.4) $)$ that $r_{\alpha}(J e+i 0)$ has the asymptotic form, coinciding with the r.h.s. of (A.7), and, as a result, we obtain that

$$
\nu_{\alpha}(J e)=\pi^{-1} \Im r_{\alpha}(J e+i 0)=\frac{v^{2}\left(1+O\left(J^{-1}\right)\right)}{J^{2} x^{2}} \nu_{-\alpha}(J e)+\nu_{0}(J e) e^{\xi_{s \alpha}}\left(1+O\left(J^{-1}\right)\right),
$$

where the first and the second terms in the r.h.s. are the contributions of $\pi^{-1} \Im I_{1}(J e+i 0)$ and $\pi^{-1} \Im I_{2}(J e+i 0)$ respectively and $\nu_{0}$ is given by (3.19). By combining (A.8) for $\alpha= \pm$ and (A.6), we obtain

$$
\nu_{\alpha}(J e) / \nu_{0}(J e) \rightarrow \exp \left\{\left(e-e_{0}\right) s \alpha / a^{2}\right\}, \quad J \rightarrow \infty .
$$

This formula and (3.20) yield (3.17).

Furthermore, formulas (4.13), (4.4), and (4.8) imply that for any continuous function $\phi$ we have

$$
\lim _{J \rightarrow \infty} \int_{-\infty}^{\infty} \phi(e) \nu_{\alpha}\left(J e, J e^{\prime}\right) J d e^{\prime}=\phi(e),
$$

i.e. that $\nu_{\alpha}\left(J e, J e^{\prime}\right) J$ tends to $\delta\left(e-e^{\prime}\right)$ as a generalized function.

\section{Derivation of formula 7.9}

We outline here a derivation of formula (7.9) for the mean reduced density matrix of the small system in the regime (7.3) - (77.4) of almost flat density of states. By using formulas (4.10) and (17.7) for $r_{\alpha}(z)$ and $r_{\alpha}(E, z)$, we can write (17.9) as

$$
\begin{aligned}
\rho_{\alpha, \alpha}(E, t) & =-\frac{1}{(2 \pi i)^{2}} \int_{-\infty}^{\infty} \frac{e^{i t \lambda_{1}}}{\lambda_{1}-I A} d \lambda_{1} \int_{-\infty}^{\infty} e^{-i t \lambda_{2}}\left[\left(\lambda_{1}-\lambda_{2}-2 i A\right) \rho_{\alpha, \alpha}(0)-2 i A \rho_{-\alpha,-\alpha}(0)\right] \\
& \times \frac{\left(\lambda_{1}-\lambda_{2}-2 i A\right)}{\left(\lambda_{1}-\lambda_{2}\right)\left(\lambda_{2}+I A\right)\left(\lambda_{1}-\lambda_{2}-4 i A\right)} d \lambda_{2} .
\end{aligned}
$$

According to (4.10), the integrand of the internal integral in $\lambda_{2}$ is the limiting value of a function that is analytic in the upper half-plane and has a cut on the real axis. We see, however, that the function can be continued in the lower half-plane and has there simple poles at $\lambda_{2}=\lambda_{1}-i 0,-i A, \lambda_{1}-4 i A$. Computing the integral by residues, we obtain after certain transformations:

$$
\rho_{\alpha, \alpha}(E, t)=\frac{1}{2 \pi i} \int_{-\infty}^{\infty} e^{i t \xi} \frac{(\xi-2 i A) \rho_{\alpha, \alpha}(0)-2 i A \rho_{-\alpha,-\alpha}(0)}{\xi(\xi-4 i A)} d \xi,
$$


where the integration path encircles $\xi=0$ from below. The integrand can be continued in the upper half-plane, and has there the simple poles at $\xi=0+i 0,4 i A$. Computing the integrals by residues, we obtain (7.9).

Our argument above is a toy version of a techniques of complex dilatation, used in [6, 1] to find the resonances (poles on the second sheet) of the Liouville operator, responsible for the exponential convergence of the density matrix of the composite system to the Gibbs distribution. Hence, the mathematical mechanism of the exponential convergence in our model is similar to that of the spin-boson and related models, studied in [6, 1].

\section{Acknowledgment}

We are grateful to V. Jacksic, T. Spencer and H. Spohn for helpful discussions. The work was supported in part by NSF Grant DMR 01-279-26, AFOSR Grant 49620-01-1-0154. We also acknowledge the hospitality of the Institute for Advance Study and of the H. Poincare Institute where part of this work was done.

\section{References}

[1] Bach V, Fröhlich J and Sigal I M 1995 Return to equilibrium Lett. Math. Phys. 34 183

[2] Bulgac A, de Dong G and Kuznetsov D 1998 Dynamics of a simple system in a complex environment Phys. Rev. E58 196

[3] Davies E B Quantum Theory of Open Systems 1976 (New York: Academic Press)

[4] Dembo A and Zeitouni O 1998 Large Deviation Techniques and Applications (Berlin: Springer)

[5] Feynman R and Vernon F 1963 The theory of a general quantum system interacting with a linear dissipative system Ann. of Phys. 24118

[6] Jaksic V and Pillet C - A 1997 Spectral theory of thermal relaxation J. Math. Phys. 381757

[7] Haake F 1973 Statistical Treatment of Open System by Generalized Master Equation (Berlin: Springer)

[8] Khorunzhy A, Khoruzhenko B and Pastur L 1996 Asymptotic properties of large random matrices with independent entries J. Math. Phys. 375033

[9] Kubo R, Toda M and Hashitsume U 1991 Statistical Physics II. Non-equilibrium Statistical Mechanics (New York: Springer)

[10] Lifshitz I, Gredeskul S and Pastur L 1988 Introduction to the Theory of Disordered Systems (New York: Wiley)

[11] Ligget A, Chakravarty S, Dorsey A T, Fisher M P A, Gorg A, and Zweiger W 1980 Dynamics of the dissipative two-state systems. Rev. Mod. Phys. 591 
[12] Lutz E and Weidenmuller H A 1999 Universality of quantum Brownian motion Physica $\mathbf{A 2 6 7} 354$

[13] Mehta M 1991 Random Matrices (New York: Academic Press)

[14] Mello P A, Pereyra P and Kumar N 1988 A soluble random-matrix model for relaxation in quantum systems J. Statist. Phys. 5177

[15] Pastur L. 1999 Mathematical Results in Statistical Mechanics eds Miracle-Sole S, Ruiz J and Zagrebnov V (Singapore: World Scientific) 429

[16] Pereyra P 1991 Random matrix model for dissipative two-level systems J. Statist. Phys. 65773

[17] Spohn H 1980 Kinetic equations from Hamiltonian dynamics: Markovian limits Rev. Mod. Phys. 52569 